\title{
EDITORIAL
}

\section{A special issue on Photonics Research in Canada}

\author{
Jianping YAO (ه) $)^{1}$, Lawrence R. CHEN $(\bowtie)^{2}$ \\ 1 School of Electrical Engineering and Computer Science, University of Ottawa, Ottawa, ON, K1N 6N5, Canada \\ 2 Department of Electrical and Computer Engineering, McGill University, Montreal, QC, H3A 0E9, Canada
}

(C) Higher Education Press and Springer-Verlag GmbH Germany, part of Springer Nature 2018

Canada has a long standing history of contributions to the field of photonics. Photonics research occurs at a number of universities across the country as well as in government research labs. The Canadian photonics industry includes small start-ups to large scale corporations, many of which are involved in research. It is estimated that photonics is a $\$ 6$ billion industry in Canada that employs more than 24000 people. Photonics clusters can be found in Vancouver, Toronto, Ottawa, Montreal, and Quebec City - cities that are also home to universities where world renowned photonics research takes place.

In this special issue, we present a highlight of recent research activities in Canada. Prominent researchers across the country have contributed papers on topics ranging from optical and wireless communications, fiber and integrated technologies, and quantum photonics.

Prof. Xiupu Zhang from Concordia University reviews the development of broadband linearization techniques, both optical and electrical, for the fronthaul transmission technologies that are crucial to emerging 5G networking. Profs. Julian Cheng and Jonathan F. Holzman from the University of British Columbia, along with their colleagues, describe optical indoor positioning systems for visible light communications. Positioning systems are a critical aspect of optical-wireless or free-space optical communications. Prof. John C. Cartledge from Queen's University provides an overview of research on coherent optical fiber communications. In particular, he describes work aimed at compensation of transmission impairments and understanding limitations on the performance of systems operating at $1 \mathrm{~Tb} / \mathrm{s}$.

Prof. Roberto Morandotti from the Institut National de la Recherche Scientifique - Centre Énergie, Matériaux et Télécommunications and his colleagues provide an overview of on-chip optical frequency combs for generating quantum states and the use of standard telecommunications components for their coherent control. The results can have significant impact on integrated photonics for entangled photon states for scalable quantum information. Prof. Li Qian from the University of Toronto and her co-authors discuss how to restore the interference visibility of a photon pair in a Franson interferometer. This work is significant as it points to methods for encoding high-dimensional quantum information in the time domain.

Profs. Leslie A. Rusch and Sophie LaRochelle from the Université Laval describe recent demonstrations of fiber transmission using orbital angular momentum modes and linearly polarized vector modes in ring core fibers. These fibers and transmission strategies offer an alternate approach for exploiting the spatial domain for optical transmission. Prof. Lukas Chrostowski from the University of British Columbia and Prof. José Azaña from the Institut National de la Recherche Scientifique - Centre Énergie, Matériaux et Télécommunications, as well as their collaborators, provide a comprehensive overview of integrated microwave photonics, and in particular, the use of waveguide Bragg gratings for microwave signal processing. Prof. Raman Kashyap from the École Polytechnique de Montréal and his collaborators describe recent achievements on using rare-earth doped materials to implement optical refrigeration, in particular, demonstration of laser cooling in high purity glass ceramics may pave the way for massive application of optical refrigeration.

We thank the publisher for the opportunity to coordinate this special issue and would like to thank the authors for contributing their articles.

Received May 3, 2018

E-mails: jpyao@uottawa.ca, lawrence.chen@mcgill.ca 


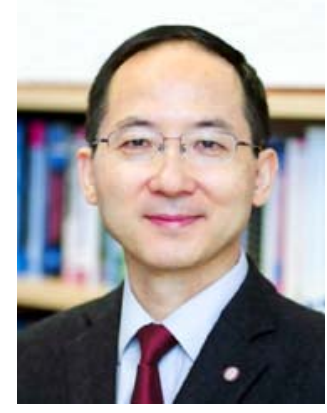

Jianping Yao is a Distinguished University Professor and University Research Chair in the School of Electrical Engineering and Computer Science, University of Ottawa, Ontario, Canada. He is a Fellow of the IEEE, the Optical Society of America, and the Canadian Academy of Engineering. He served as Director of the Ottawa-Carleton Institute for Electrical and Computer Engineering 2007-2010 and 2013-2016. During his career, he has authored over 560 papers including over 330 in refereed journals and 230 in conference proceedings. Currently he serves as Chair of the IEEE Ottawa Photonics Chapter and the Technical Committee Chair of IEEE MTT-3 Microwave Photonics. He is Editor-in-Chief of IEEE Photonics Technology Letters, a former Topical Editor of Optics Letters, an Associate Editor of Science Bulletin, and an Advisory Editorial Board member of Optics Communications. He served as a chair or co-chair of multiple international conferences including the General Cochair of the 2017 IEEE International Topical Meeting on Microwave Photonics and General Chair of the 2019 IEEE International Topical Meeting on Microwave Photonics. Prof. Yao was an IEEE MTT-S Distinguished Microwave Lecturer for 2013-2015. He was the recipient of the Award for Excellence in Research of the University of Ottawa 2017-2018. In 2018, he received the R.A. Fessenden Silver Medal from IEEE Canada.

E-mail: jpyao@uottawa.ca

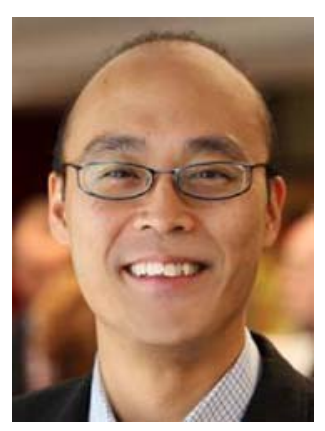

Lawrence R. Chen received the B.Eng. degree in electrical engineering and mathematics from McGill University, Montreal, QC, Canada, in 1995 and the M.A.Sc. and Ph.D. degrees in electrical and computer engineering in 1997 and 2000, respectively. Since 2000, he has been with the Department of Electrical and Computer Engineering, McGill University. His research interests include optical communications, fiber and integrated optics, and microwave photonics (in particular, active and passive devices in silicon photonics for optical and microwave signal processing) as well as engineering education and teaching pedagogy.

Prof. Chen is an Editor for Optics Communications and a Subject Editor for IET Electronics Letters. He is a former Editor-in-Chief of the IEEE Photonics Newsletter and a former Topical Editor of Optics Letters. Prof. Chen has served on the program committees for a number of international conference and was Chair of the IEEE Photonics Conference Subcommittee on Optical Networks and Systems (2009-2011); he will be the Technical Program Chair for the 2019 IEEE Topical Meeting on Microwave Photonics.

Prof. Chen is a professional engineer in the province of Quebec and is a Fellow of the Optical Society of America (OSA).

E-mail: lawrence.chen @mcgill.ca 